\title{
Surgical removal of a compressive thoracic epidural vascular malformation in a patient with Klippel-Trénaunay syndrome: illustrative case
}

\author{
John C. Muse, MD, Luke Antonio Silveira, MD, Brandon Liebelt, MD, and Bruce lan Tranmer, MD \\ Department of Neurosurgery, University of Vermont, Burlington, Vermont
}

BACKGROUND Klippel-Trénaunay syndrome (KTS) is a combined capillary-lymphatic-venous malformation disorder traditionally associated with high surgical morbidity. Although rare, pathologic involvement of the spinal cord has been reported in the literature. However, the safety of surgical intervention remains unclear. We report a case of successful decompression of a thoracic epidural lesion in an individual with KTS who presented with spastic paraparesis.

OBSERVATIONS The patient is a 38-year-old male, diagnosed with KTS as an infant, who presented with spastic paraparesis secondary to a thoracic epidural lesion. He underwent laminectomies and resection of the lesion with subsequent improvement of his symptoms and without significant postoperative morbidity. Histopathology confirmed the lesion to be a benign vascular malformation.

LESSONS Currently, the literature regarding management of symptomatic vascular lesions in individuals with KTS supports nonoperative management, due to the increased risk of operative morbidity associated with this syndrome. This case presents evidence for safe and appropriate surgical management of a thoracic epidural vascular malformation in a patient with KTS in the setting of progressive neurological decline, establishing a role for neurosurgical intervention in this high-risk population when no conservative management portends further neurological deterioration.

https://thejns.org/doi/abs/10.3171/CASE21634

KEYWORDS Klippel-Trénaunay syndrome; thoracic; vascular malformation

Klippel-Trénaunay syndrome (KTS) is a rare, multifactorial disorder that is characterized by vascular malformations effecting the capillary, venous, and lymphatic systems. It is associated with three cardinal features: cutaneous capillary malformations, venous malformations, and soft tissue and/or bony hypertrophy of the extremities. ${ }^{1,2}$ Individuals with this syndrome often suffer from complications relating to vascular and/or lymphatic malformations that predispose them thrombophlebitis, cellulitis, deep venous thrombosis, and internal hemorrhage owing to abnormal vessels supplying internal organs. ${ }^{1,3}$ Management of symptomatic vascular malformations remains largely nonsurgical, as there are numerous reports of poor wound healing and difficulty achieving hemostasis in patients with KTS. ${ }^{4,5}$ Although uncommon, various spinal pathologies have been documented in some individuals with presumed KTS, including spinal arteriovenous malformations, spinal cavernous angiomas, vertebral hemangiomas, scoliosis, paravertebral tumors, and extradural meningeal cysts. ${ }^{6-11}$ To date, there is only one report of an epidural lesion causing spinal cord compression in a patient with KTS. ${ }^{10}$ We present a case of a thoracic epidural lesion causing spastic paraparesis in a patient with KTS that was successfully resected and resulted in symptomatic improvement. The purpose of this illustrative case is to outline to measures taken to minimize morbidity while performing a necessary neurosurgical intervention on a high-risk surgical patient with a rare disease, KTS.

\section{Illustrative Case}

\section{Presentation}

A 38-year-old male, initially diagnosed with lymphangiomatosis at birth, was later more specifically diagnosed with KTS. Lifetime sequelae of his condition included hypertrophy of his right upper extremity and left lower extremity, lymphangiomatosis of his internal organs, cutaneous nevi, and multiple subcutaneous lymphatic cysts, some of which had required operative excision. One such lesion on his right

ABBREVIATIONS CLVM = capillary-lymphatic-venous malformation; KTS = Klippel-Trénaunay syndrome; MRI = magnetic resonance imaging INCLUDE WHEN CITING Published January 3, 2022; DOI: 10.3171/CASE21634.

SUBMITTED November 4, 2021. ACCEPTED November 17, 2021.

(C) 2022 The authors, CC BY-NC-ND 4.0 (http://creativecommons.org/licenses/by-nc-nd/4.0/). 


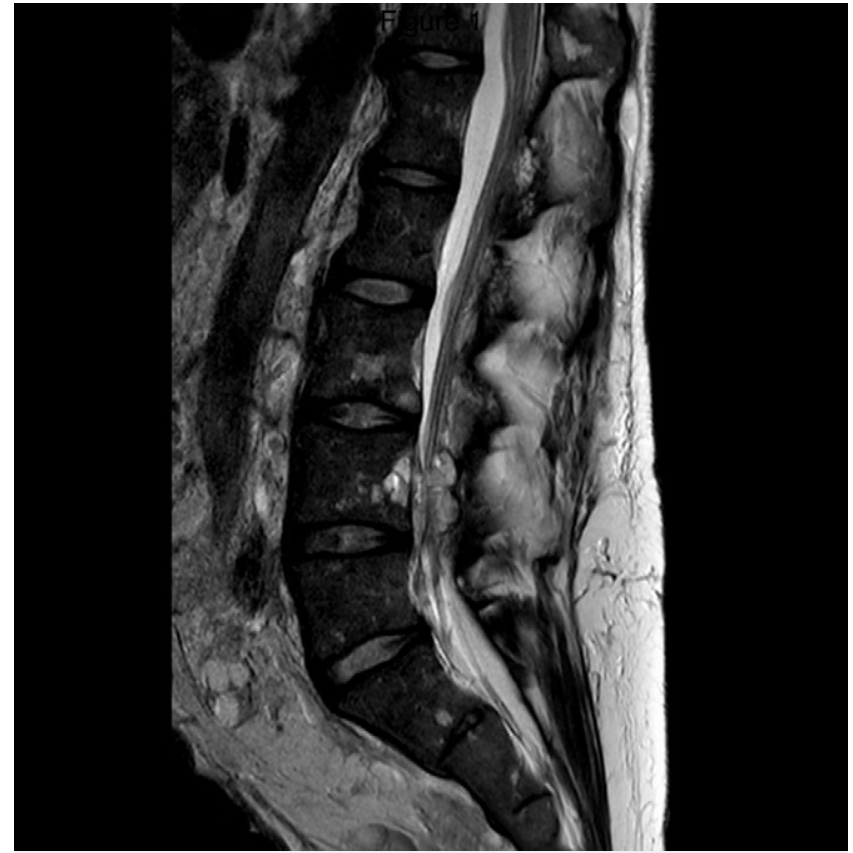

FIG. 1. Sagittal T2-weighted MRI. Midlumbar stenosis secondary to compression from abnormal, presumed lympho-vascular, tissue.

flank demonstrated persistent weeping since his operation many years ago. Despite the complications of his condition, the patient lived an independent life and worked as a professor at a local university.

At age 36, the patient first presented with buttock pain that radiated down into his right thigh. He additionally reported subjective weakness in his right leg, which resulted in difficulty ambulating. There was no associated sensory loss or involvement of his bowel or bladder. His examination at that time demonstrated an abnormal gait and diminished reflexes in his lower extremities but full strength. Magnetic resonance imaging (MRI) of his lumbar spine revealed midlumbar severe spinal stenosis due to abnormal tissue compressing the cauda equina (Fig. 1). The remainder of his spine was subsequently imaged, unrevealing for any additional areas of canal compromise. At the time, conservative management and observation was favored over surgical intervention given the concern for poor wound healing and commonly described difficulty obtaining hemostasis in patients with KTS.

At age 38, the patient developed increased subjective weakness of his right leg as well as numbness of his left leg. He noted that his right leg would give out on him, and this was significantly impacting his ability to ambulate as he newly required the assistance of a walker. The patient denied new pain, bowel, or bladder involvement. On examination, the patient had full strength in his lower extremities, but increased tone in his right lower extremity and bilateral lower extremity hyperreflexia including sustained clonus in the right lower extremity. A rectal examination revealed intact sensation with diminished resting tone and diminished voluntary contraction.

An MRI of his neuroaxis redemonstrated the known lumbar lesion, unchanged from prior, in addition to a new thoracic lesion extending from a paraspinal/posterior mediastinal lesion along the upper thoracic spine and causing severe cord compression at T2 and T3 (Fig. 2). To better characterize the nature of this lesion, he subsequently underwent spinal angiography, negative for any abnormal vascular flow to this lesion. A lymphoscintigraphy was performed, which showed lymphatic malformations in the right thigh and left pelvis as well as dilated and tortuous lymphatic vessels within the abdomen. Unfortunately, there was minimal radiotracer uptake in the chest, which suggested that the lymphatics of the lower extremity terminated intra-abdominally.

\section{Operation of Interest and Postoperative Course}

The patient was noted to have increasing difficulty with ambulation secondary to lower extremity weakness. This was transiently responsive to treatment with Decadron. However, due to his progressive weakness, it was felt that he warranted surgical decompression of the thoracic lesion. He underwent bilateral thoracic laminectomies at T2 and T3 with intraoperative neurophysiological monitoring. Numerous abnormities were encountered during surgery including infiltration of his paravertebral muscles with hemangiomatous tissue and trabeculated vascular bone in his lamina. Removal of the lamina at T2 and T3 revealed large venous varices in the epidural space, which passed through the neural foramina at T2 and T3 on the right side and entered the chest cavity. The tissue itself was noted to be hemangiomatous-like with large venous channels (Fig. 3). Bipolar cautery was used to open the venous channels and shrink the varices, resulting in wide decompression. Despite extensive bleeding, hemostasis was obtained using Gelfoam and meticulous bipolar cautery. Somatosensory-evoked potentials (SSEPs) and motor-evoked potentials (MEPs) did not change significantly during the procedure. Tissue biopsied during the procedure was sent for pathology. Immunohistochemistry revealed anastomosing ectatic vessels lined by bland endothelial cells, consistent with a benign vascular malformation.
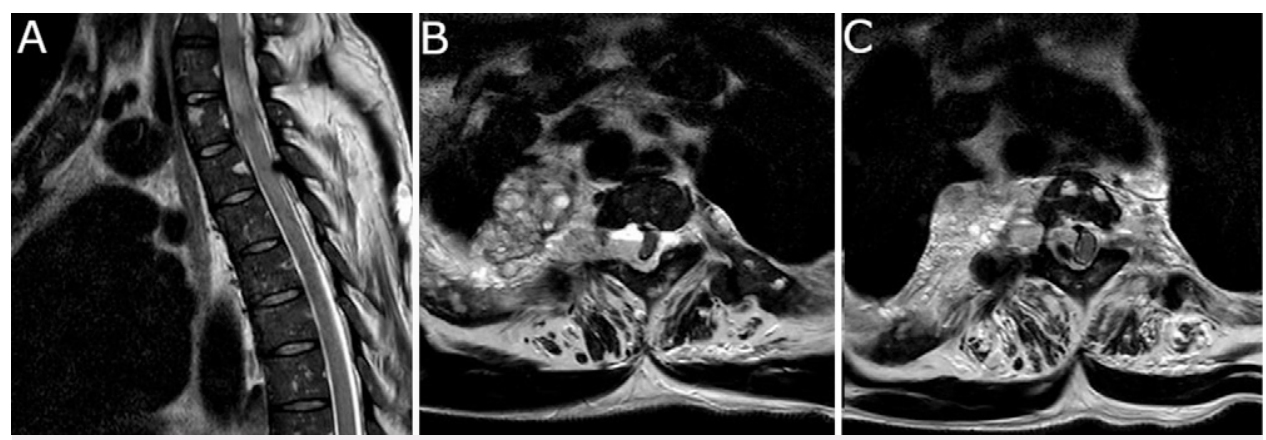

FIG. 2. Sagittal (A) and axial (B and C) T2-weighted MRI. Thoracic stenosis at T2 (B) and T3 (C) secondary to compression from abnormal tissue that extends from right mediastinal region into spinal canal. 


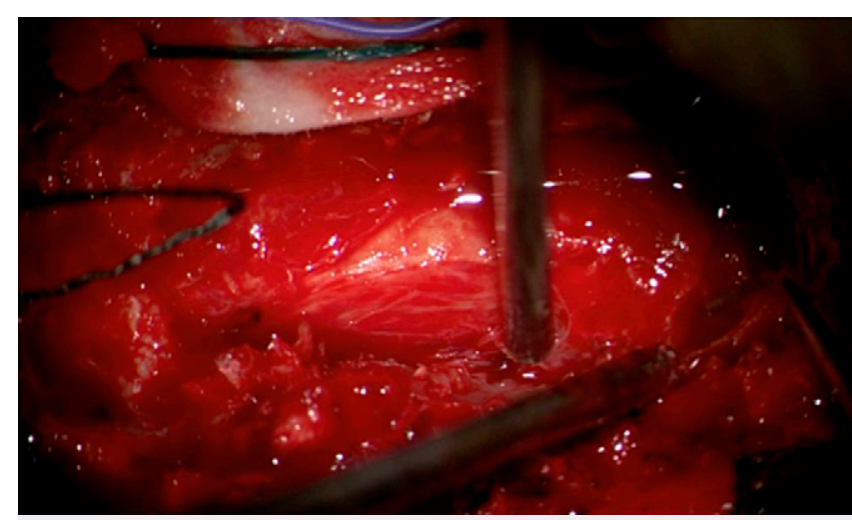

FIG. 3. Intraoperative image of abnormal compressive tissue encountered during thoracic laminectomies and decompression at T2 and T3. Tissue was noted to be hemangiomatous-like with large venous channels.

The patient was discharged home uneventfully on postoperative day 4. At his 1-month postoperative visit, he reported significant improvement in his lower extremity strength and numbness and stated that he only occasionally needed the assistance of a walker to ambulate. At that time an MRI showed resolution of the compressive lesion at the T2 and T3 levels (Fig. 4). At his 6-month postoperative visit, his gait was essentially normal, and he reported no new symptoms.

\section{Discussion}

\section{Observations}

Originally described in 1900, KTS is a combined capillary-lymphatic-venous malformation (CLVM) without associated high flow arteriovenous fistulas. ${ }^{1,12}$ Despite KTS being a recognized syndrome for more than a century, its etiology has yet to be fully elucidated and its diagnosis can be challenging. In 2014, the International Society for the Study of Vascular Anomalies classified KTS as one of the vascular malformations associated with other anomalies. $^{13}$ Specifically, the inclusion of limb hypertrophy (overgrowth) was included as a criteria for its classification in addition to CLVM. Despite much investigation, no causal genetic link has yet to be identified, and it is currently felt that pathogenesis of this condition is polygenetic in nature. ${ }^{14}$ Diagnosis is made clinically with variable symptoms between patients owing to the variable anatomical locations of vascular lesions. Of note, there is a need to differentiate KTS from the closely related syndrome known as Parkes-
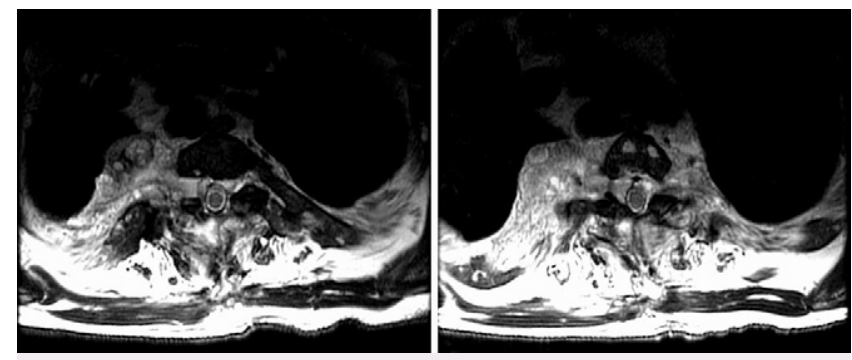

FIG. 4. Postoperative thoracic axial T2-weighted MRI. Resolution of thoracic stenosis at T2 (left) and T3 (right). Persistence of abnormal tissue in the right mediastinal region.
Weber syndrome (analogous to Klippel-Trénaunay-Weber syndrome), which is distinguished from KTS by the presence of at least one hemodynamically significant arteriovenous fistula and whose treatment and prognosis vary significantly from KTS.

The heterogeneity in clinical manifestation of KTS has precluded any clear guidelines for management of the sequala of this syndrome from fully being established. This is especially true in the case of compressive spinal lesions, which are exceedingly rare in this patient population. Currently, there are basic guidelines for the management of vascular malformations in patients with KTS, the general tenants of which advocate nonoperative management as primary treatment. These include lifestyle modification, wound care, orthotics, and compression therapy. ${ }^{4}$ Those with progressive symptoms should be evaluated for minimally invasive therapies including pulsed laser therapy, embolization, sclerotherapy, and radiofrequency or laser ablation. Multiple reports of persistent bleeding and poor wound healing in patients with KTS have resulted in open surgical treatment being considered a last resort therapy. ${ }^{1,4,5}$ These guidelines are primarily geared toward the management of the more common complications of KTS relating to chronic venous insufficiency and outflow.

Due to its rare occurrence, there are no guidelines for the management of vascular malformations causing spinal cord compression in patients with KTS. Indeed, there are only two additional case reports of compressive spinal lesions in patients with reported KTS. Alexander et al. ${ }^{6}$ reported a case of a 30-year-old male with KTW syndrome who presented with progressive lower extremity weakness and numbness in whom an extradural thoracic AVM was discovered and successfully removed. In 1995, Carter et al. ${ }^{10}$ reported a 20 -year-old male with a diagnosis of KTW syndrome who presented with progressive lower extremity weakness and urinary retention in whom a thoracic extradural angiomyolipoma was successfully removed with subsequent improvement in his neurological function. Although similar in presentation and both requiring operative intervention, it should be noted that in the case reported by Alexander et al. ${ }^{6}$ the patient does not meet the current criteria for KTS given the angiographically confirmed AVM that was surgically excised. While Carter et al. ${ }^{10}$ described resection of an angiomyolipoma, the patient described in this case had resected tissue consistent with a benign vascular malformation on pathologic analysis.

\section{Lessons}

Here, we present a case of an individual with KTS who successfully underwent thoracic laminectomies and decompression of a compressive epidural vascular malformation with resulting improvement of his lower extremity function. Furthermore, there were none of the feared complications associated with open surgical treatment of patients with KTS, namely uncontrollable bleeding or poor wound healing. This case provides evidence that patients with KTS who have compressive vascular malformations in the spinal canal can safely and appropriately be treated with surgical intervention.

\section{References}

1. Sung HM, Chung HY, Lee SJ, et al. Clinical experience of the Klippel-Trenaunay syndrome. Arch Plast Surg. 2015;42(5):552-558.

2. Jacob AG, Driscoll DJ, Shaughnessy WJ, Stanson AW, Clay RP, Gloviczki P. Klippel-Trénaunay syndrome: spectrum and management. Mayo Clin Proc. 1998;73(1):28-36.

3. Billington AR, Shah J, Elston JB, Payne WG. Klippel-Trenaunay syndrome. Eplasty. 2013;13:ic64. 
4. Wang SK, Drucker NA, Gupta AK, Marshalleck FE, Dalsing MC. Diagnosis and management of the venous malformations of KlippelTrénaunay syndrome. J Vasc Surg Venous Lymphat Disord. 2017;5(4):587-595.

5. Noel AA, Gloviczki P, Cherry KJ Jr, Rooke TW, Stanson AW, Driscoll DJ. Surgical treatment of venous malformations in KlippelTrénaunay syndrome. J Vasc Surg. 2000;32(5):840-847.

6. Alexander MJ, Grossi PM, Spetzler RF, McDougall CG. Extradural thoracic arteriovenous malformation in a patient with Klippel-Trenaunay-Weber syndrome: case report. Neurosurgery. 2002;51(5): 1275-1278, discussion 1278-1279.

7. Oda K, Morimoto D, Kim K, Yui K, Kitamura T, Morita A. Spinal cavernous angioma associated with Klippel-Trenaunay-Weber syndrome: case report and literature review. World Neurosurg. 2018;109:333-337.

8. Okutan O, Yildirim T, Isik S, Gokce B, Saygili B, Konakli EB. Thoracic vertebral hemangioma causing paraplegia in KlippelTrenaunay-Weber syndrome: case report. Turk Neurosurg. 2013; 23(4):518-520.

9. Choi KC, Ahn ST, Shin YH, Lee SH. Spinal extradural meningeal cyst in Klippel-Trenaunay syndrome. J Korean Neurosurg Soc. 2011;49(5):299-301.

10. Carter DA, Kim K, Brinker RA. Extradural tumor causing spinal cord compression in Klippel-Trenaunay-Weber syndrome. Surg Neurol. 1995;43(3):257-260.

11. Ouellet MC, Sevick RJ, Tranmer BI, Lester WM. Thrombosed fusiform basilar aneurysm associated with Klippel-Trenaunay-Weber syndrome: case report. Can Assoc Radiol J. 1997;48(1):28-32.
12. Wassef M, Blei F, Adams D, et al. Vascular anomalies classification: recommendations from the International Society for the Study of Vascular Anomalies. Pediatrics. 2015;136(1):e203-e214.

13. McCuaig CC. Update on classification and diagnosis of vascular malformations. Curr Opin Pediatr. 2017;29(4):448-454.

14. Oduber CE, van der Horst CM, Hennekam RC. Klippel-Trenaunay syndrome: diagnostic criteria and hypothesis on etiology. Ann Plast Surg. 2008;60(2):217-223.

\section{Disclosures}

The authors report no conflict of interest concerning the materials or methods used in this study or the findings specified in this paper.

\section{Author Contributions}

Conception and design: Muse, Tranmer. Acquisition of data: Tranmer. Drafting the article: Silveira, Muse, Tranmer. Critically revising the article: Silveira, Liebelt, Tranmer. Reviewed submitted version of manuscript: all authors. Approved the final version of the manuscript on behalf of all authors: Silveira.

\section{Correspondence}

Luke Antonio Silveira: University of Vermont, Burlington, VT. luke. silveira@uvmhealth.org. 